\title{
ATTACK PRIMING IN FEMALE SYRIAN GOLDEN HAMSTERS IS ASSOCIATED WITH A c-fos-COUPLED PROCESS WITHIN THE CORTICOMEDIAL AMYGDALA
}

\author{
M. POTEGAL, ${ }^{*} \dagger$ C. F. FERRIS, $\ddagger$ M. HEBERT, ${ }^{*}$ J. MEYERHOFF* and \\ L. SKAREDOFF* \\ *Department of Medical Neurosciences, Walter Reed Army Institute of Research, Washington, \\ DC 20307-5100, U.S.A. \\ $\ddagger$ Department of Psychiatry, University of Massachusetts Medical Center, Worcester, MA 01605 , \\ U.S.A.
}

\begin{abstract}
Allowing a resident hamster a single "priming" attack on a conspecific induces a transient aggressive arousal as indicated by a reduction in the latency and increase in the probability of attack on a second intruder presented within the next $30 \mathrm{~min}$. We present two lines of evidence identifying the corticomedial amygdala as an important locus mediating this effect. (1) Attack priming significantly increases the number of neurons expressing immunocytochemically identified Fos protein in the corticomedial amygdala, but not elsewhere. Pursuit and biting of an inanimate object does not induce corticomedial amygdala c-fos expression of the same pattern or magnitude. The corticomedial amygdala contribution to the priming effect involves more than a non-specific arousal, since corticomedial amygdala c-fos expression does not correlate with locomotor activity, a standard indicator of such arousal. (2) Radiofrequency lesions of the corticomedial amygdala reduce aggression, the greatest reduction occurring with the more anterior lesions. Other behaviors, including a priming-like locomotor practice effect in a running wheel, are unaffected by corticomedial amygdala lesions. These findings suggest that attack priming is an aggression-specific effect resulting from a Fos-coupled change within neural circuitry of which the corticomedial amygdala is a part.

From a theoretical point of view, these experiments suggest a new approach to the analysis of the mechanisms underlying aggressive behavior and the persistence of aggressive arousal. We present a sketch of a quantitative neurobehavioral model which relates attack probability to neural activation within the corticomedial amygdala. From a methodological viewpoint, these experiments extend the utility of mapping c-fos expression as a technique for localizing endogenous, behavior-specific processes within the central nervous system. Copyright (C) 1996 IBRO. Published by Elsevier Science Ltd.
\end{abstract}

Key words: aggression, arousal, immunocytochemistry, sex, time-course.

Field and laboratory observations have revealed short-term increases in aggression during the initial stages of agonistic encounters in many species. The increased "attack readiness"19 or "aggressive arousal" 47 engendered under these circumstances can persist even after the provoking stimulus has dis-

Research was conducted in compliance with the Animal Welfare Act and other Federal statutes and regulations relating to animals and experiments involving animals, and adheres to principles stated in the Guide for the Care and Use of Laboratory Animals, NIH Publication 85-23. The views of the authors do not purport to reflect the position of the Department of the Army or the Department of Defense (Para 4-3, AR 360-5). A preliminary report on this research was presented at the Society for Neuroscience meeting, 1991.

$\dagger$ To whom correspondence should be addressed at: Department of Psychology, University of Wisconsin-Madison, 1202 W. Johnson Street, Madison, WI 53706, U.S.A.

Abbreviations: AL, amygdala level; AP, anterior-posterior; CMA, corticomedial amygdala; IRN, immunoreactive nuclei; $\mathrm{MN}$, medial nucleus. appeared. To investigate the neural bases of such persisting aggressive arousal, we first developed a laboratory model of this phenomenon within the standard resident/intruder paradigm used in the study of rodent aggression. "Attack priming" a resident hamster by allowing it a single attack on a conspecific intruder introduced into its home cage reduces the latency and increases the probability of attack on a second, "probe" intruder. ${ }^{4,52,53}$ This priming effect appears relatively specific in terms of both the responses it triggers and its effective stimuli. On the response side, we have found that other behaviors such as wheel running and eating (in female hamsters) and copulation (in male hamsters) are unaffected by priming ${ }^{53}$ (Potegal, Hebert and Meyerhoff, unpublished observations). Experiment 1 of the present report examines the specificity of the stimulus by comparing the effects following attack priming to those following the detection, pursuit and biting of an inanimate object. Other experiments have eliminated a number of alternatives to the 
aggressive arousal interpretation of attack priming, including the possibility that it is an instance of non-specific arousal. ${ }^{45}$

The priming paradigm allows investigation of the internal mechanisms of aggressive arousal by controlling the external stimulus situation. Thus, the intruders used in these studies have been group housed to suppress their aggressiveness and to create a shared cage odor. ${ }^{7,29}$ They were also routinely pretreated with the analgesic-sedative methotrimeprazine, further reducing the variability within and between intruders. These efforts at standardization have proven successful in that there is no indication that subjects discriminate among individual intruders. ${ }^{45}$ This intended result rules out explanations of attack priming as a novelty effect (i.e. dependent upon a perceived difference between the first and second intruders). This result also implies that the reduction in attack latency from priming trial to probe trial must be internally mediated. Further evidence for internal mediation has been developed by interpolating different delay intervals between the priming and probe trials. These studies show that attack latencies remain reduced for at least $30 \mathrm{~min}$ after priming. ${ }^{44,46,52}$ Finally, we note that the priming effect is not restricted to attacks on drug-treated intruders; priming two subjects before they are placed in the same arena hastens the onset of fighting between them. ${ }^{53}$ In short, attack priming induces a transient, relatively specific, internally mediated increase in aggressive arousal that generalizes across several different situations.

The behavioral characterization of attack priming sets the stage for investigation into the brain mechanisms of aggressive arousal. The relative simplicity of the paradigm and the fact that a significant shift in behavior is signaled by a brief event are properties well suited to the detailed correlation of aggressive behavior characteristics and brain mechanisms. The suitability of the paradigm can be illustrated by contrasting the priming paradigm with the standard procedure of allowing subjects to fight for a fixed period and then preparing them for neurochemical assay (e.g., Ref. 43). Because the significance of the fixed period of fighting for each individual animal is not generally established beforehand, it remains unclear if the neurochemical data are related to the actual duration of fighting, the relative pain and stress experienced during the fight, the fatigue resulting from the fight, the subsequent probability of fighting or fleeing, or simply the metabolic requirements of a period of vigorous activity.

Our general approach using the priming paradigm has been to first identify neural processes associated with aggressive arousal by using a marker of endogenous activity. Experimental manipulations, i.e. lesions or stimulation, are introduced in the second stage. The logic of this approach is that assay-type techniques are a more appropriate starting point for identifying behavior-relevant neural loci and pro- cesses because the labeling of endogenous processes, particularly those which can be histochemically assayed in whole brain sections, is a less restrictive way of identifying loci of interest than are experimental interventions which require the investigator to define and select the site (or sites) of manipulation beforehand. When such manipulations alter behavior, moreover, they can do so by affecting a multitude of processes which are neither intrinsic nor specific to that behaviour. However, while finding associations between behaviors and endogenous neural processes is arguably the best starting point, it is only a starting point. Processes may be labeled which are epiphenomenal to, secondary to or even compensatory to those actually controlling the behaviors in question. Experimental interventions are an appropriate and necessary second step in the evaluation of candidate loci and processes which have been identified previously by assay techniques.

Following this design logic, we asked whether the priming effect is mediated by neural circuitry involving the corticomedial amygdala (CMA). The marker we chose was expression of the c-fos gene; immunocytochemical mapping of Fos protein has proven informative in localizing brain regions involved in other behaviors (e.g., Refs 2, 28 and 54). Experiment 2 compares c-fos expression in the brains of attack primed subjects to that in subjects which have detected, pursued and bitten an inanimate object. These sensorimotor acts are similar to those of intraspecific attack, but do not induce aggressive arousal. Experiment 3 takes the converse approach by evaluating c-fos expression associated with a subset of the cues occurring in attack priming, cues which are known to induce a degree of aggressive arousal. This experiment is based on our earlier observations that aggressive resident hamsters will explore but will not attack a conspecific intruder which has been anesthetized to the point of immobility. ${ }^{49}$ However, the subjects' latencies to attack a subsequently presented ambulatory intruder was systematically reduced by increasing periods of pre-exposure to the anesthetized intruder. Subjects which explored an anesthetized intruder for $60 \mathrm{~s}$ subsequently attacked almost, but not quite, as quickly as when they had been fully attack primed. Experiment 4 of the present report is a lesion study probing the functional significance of the Fos localization obtained in Experiments 2 and 3.

\section{EXPERIMENTAL PROCEDURES}

\section{Subjects and intruders}

Subjects were mature, $120-180 \mathrm{~g}$ female golden hamsters (Charles River Laboratories) which were ovariectomized under $80 \mathrm{mg} / \mathrm{kg}$ pentobarbital (Nembutal) anesthesia and isolated in large polycarbonate cages, as in our previous studies (e.g., Ref. 53). Food and water were available ad libitum. At least two weeks after ovariectomy, subjects were screened for aggressiveness by having a methotrimeprazinetreated intruder (see below) placed in their home cages for 10 min. An attack was scored when a subject performed a species-typical bite and kick movement ${ }^{14}$ and/or when the 
intruder squealed and struggled during physical contact with the subject. All subjects selected for these studies attacked the intruder at least once. It should be noted that the incidence of fighting in ovariectomized females is not different from that of intact females on non-estrous days. ${ }^{12,16,63}$

Intruders were group-housed, $60-100 \mathrm{~g}$ gonadally intact female hamsters from the same supplier. These animals were routinely injected before use with the long-acting analgesicsedative methotrimeprazine (Levoprome, Lederle; 2-6 mg/ $\mathrm{kg}$, i.m.), which reduces the stress of being attacked by raising pain thresholds. ${ }^{48}$ The drug also eliminates the wild flight that some intruders show after being attacked. Treated animals continue to locomote, however. Methotrimeprazine thus induces behavior which is adequate for the elicitation of aggression, is reasonably stable from trial to trial, and is consistent across intruders. ${ }^{45}$ The validity and reliability of these intruders as elicitors of intraspecific aggression have been demonstrated in our previous studies. ${ }^{48,52}$ Subjects and intruders were maintained under a reversed 10 -h dark/14-h light cycle.

\section{Immunocytochemistry}

Subjects were anesthetized with sodium pentobarbital ( $250 \mathrm{mg} / \mathrm{kg}$, i.p.; Nembutal, Abbott) and perfused through the heart with $4 \%$ paraformaldehyde in $0.1 \mathrm{M}$ phosphatebuffered saline ( $\mathrm{pH} 7.4)$ delivered with a syringe pump. The brains were postfixed in paraformaldehyde for $1 \mathrm{~h}$, then transferred to $30 \%$ sucrose in phosphate-buffered saline. Coronal sections were cut frozen at $50 \mu \mathrm{m}$ one to two days later; alternate sections through the hypothalamus and amygdala were saved. The sections from a given brain were loaded into one of several cylindrical 30-ml incubation chambers fitted with a nylon mesh filter above a release valve at the bottom. Sections from triplets (Experiment 2) or pairs (Experiment 3 ) of subjects were run simultaneously in these chambers using aliquots of the same solutions. All incubation and rinse steps except the first and last were carried out in these chambers. The incubation sequence was: (i) $15 \mathrm{~min}$ in $0.3 \% \mathrm{H}_{2} \mathrm{O}_{2}$ to eliminate endogenous peroxidases; (ii) $30 \mathrm{~min}$ in $10 \%$ normal goat serum with $0.2 \%$ Triton X-100 at $4^{\circ} \mathrm{C}$; (iii) overnight in $0.2 \mu \mathrm{g} / \mathrm{ml}$ primary antibody (1:500 dilution of rabbit polyclonal antibody Ab-2; Oncogene Sciences, Manhassett, NY, U.S.A.) at $4^{\circ} \mathrm{C}$ with $2 \%$ normal goat serum and $0.1 \%$ bovine serum albumin as non-specific binding blockers; (iv) $45 \mathrm{~min}$ in $1 \mu \mathrm{g} / \mathrm{ml}$ biotinylated goat anti-rabbit immunoglobulin $G$ (Vector Laboratories, Burlingame, CA, U.S.A.); (v) $30 \mathrm{~min}$ in avidin/biotinylated horseradish peroxidase $\mathrm{H}$ complex (Vectastain Elite ABC Kit, Vector Laboratories); the sections were copiously rinsed between steps; (vi) $0.025 \%$ pre-filtered 3,3'-diaminobenzidine in $0.1 \mathrm{M}$ acetate buffer as the primary chromogen and $2.5 \% \mathrm{NiNH}_{2} \mathrm{SO}_{4}$ as an enhancer; ${ }^{17} \mathrm{H}_{2} \mathrm{O}_{2}$ was added just before incubation for a final concentration of $0.0125 \%$. A group of randomly chosen sections was monitored in their reaction dish under a dissecting microscope until the nuclei become visible (usually $3-5 \mathrm{~min}$ ); the reaction was terminated by immersion in $0.1 \mathrm{M}$ acetate buffer. Tests for specificity were: (i) omission of the primary antibody; (ii) substitution of normal rabbit immunoglobulin $\mathbf{G}$ for the primary; (iii) preadsorption of the primary antibody with $1.0 \mu \mathrm{g} / \mathrm{ml}$ of Fos protein (Oncogene Science). All tests eliminated nuclear staining. Sections were lightly counterstained with Toluidine Blue after mounting for identification of structures.

To obtain initial, qualitative pictures of the distribution of Fos immunoreactive nuclei (IRN), a computer-aided brain mapping system ${ }^{27}$ was used to create video images of brain sections in which the background was faded until only Fos-stained nuclei were visible. Images of homologous sections from similarly treated subjects were superposed to form composite images; for the case of the amygdala reported here, the individual images were aligned along the ventral surface of the temporal lobe. Follow-up, quantitative analysis involved counting Fos IRN at $\times 150$ using a concentric circle reticule (Bausch and Lomb $3163 \mathrm{~TB}$ ). Bilateral Fos IRN counts were taken at four coronal amygdala levels (ALs), defined by the following white matter landmarks: ALl, the caudal margin of the optic chiasm; AL2, where a line drawn through the middle of the fornices bisected the optic tract dorsoventrally; AL3, where a line through the fornices coincided with the ventral edge of the optic tract; AL4, where the ventral edges of the cerebral peduncle and the optic tract coincided. These levels are roughly $350 \mu \mathrm{m}$ apart. Counts of the medial nucleus (MN) at AL1-AL3 included all stained nuclei within three quadrants of a reticule-defined circle $(1 \mathrm{~mm}$ diameter on the section) placed at a tangent to the optic tract and the ventral edge of the temporal lobe; the circle was rotated so the uncounted quadrant was lateral to the dorsal MN (see Fig. $1 B$ ). $\mathrm{MN}$ counts at AL4 were made within a $1 \mathrm{~mm}$ image diameter vertical half circle covering the medial aspect of the temporal lobe. Nuclei in the anterior cortical nucleus (C1 in Newman's nomenclature for the hamster ${ }^{25,26}$ ) at AL1-AL3 were counted within a circle quadrant just adjacent to the MN sample circle, with apex up and lower edge at a tangent to the ventral edge of the temporal lobe (Fig. 1B). The posterolateral cortical nucleus (C2 in Newman's nomenclature) was counted at AL3 and AL4, the posteromedial cortical nucleus (C3 in Newman's nomenclature) at AL4 within quadrants of an annulus and a circle, respectively. Other nuclei of the amygdala were counted within appropriately sized and positioned whole or part circles (Fig. 1B).

\section{Lesions}

Subjects in Experiment 4 were operated under $100 \mathrm{mg} / \mathrm{kg}$ i.p. thiopental (Pentothal, Abbot) anesthesia. Stereotaxic coordinates for the CMA lesions were: anterior-posterior (AP) $=$ bregma, lateral $=$ midline $\pm 2.9 \mathrm{~mm}$, ventral $=$ skull surface $-8.7 \mathrm{~mm}$. The same AP and lateral coordinates were used for the cortical control group, but their lesions were made $3.0 \mathrm{~mm}$ below the skull surface. The electrode was a $0.25 \mathrm{~mm}$ diameter Formvar-coated, stainless steel wire with a $0.25 \mathrm{~mm}$ uninsulated tip. For the CMA and cortical lesion groups, radiofrequency current from a Radionics RFG-4 lesion maker was raised slowly to $30 \mathrm{mils}$ to reduce cavitation. The electrode placement for the electrode track group was identical to that of the lesion group, but the electrode was withdrawn without passing current.

\section{Experimental protocols}

Experiment 1. This experiment compared the effects on subsequent attack latency of attack priming vs the pursuit and biting of an inanimate object. Twenty-one aggressive subjects were trained to pursue a hamster-sized block of wood moved on a metal armature in a back and forth horizontal arc through their cages to obtain the sunflower seeds which had been taped to it. The subjects quickly learned to follow the block and remove the seeds; they also often spent some time gnawing on the block. Each subject was then given, in ABBA or BAAB order, two priming/ probe trial pairs and two woodblock/probe trial pairs. Priming/probe trial pairs were organized as follows. On the first (priming) trial, a methotrimeprazine-treated intruder was placed into the subject's home cage and left there until the standard priming condition criterion of one biting attack was met or 10 min elapsed. The priming intruder was then withdrawn immediately, after which the probe trial began when a second intruder was presented for $10 \mathrm{~min}$. On woodblock/probe trial pairs, a 3-min exposure to the moving, seed-loaded woodblock was substituted for the priming trial. The total time during which the subject was in contact with, or within half a body length of, the intruder before the attack on priming trials was recorded; identical contact 

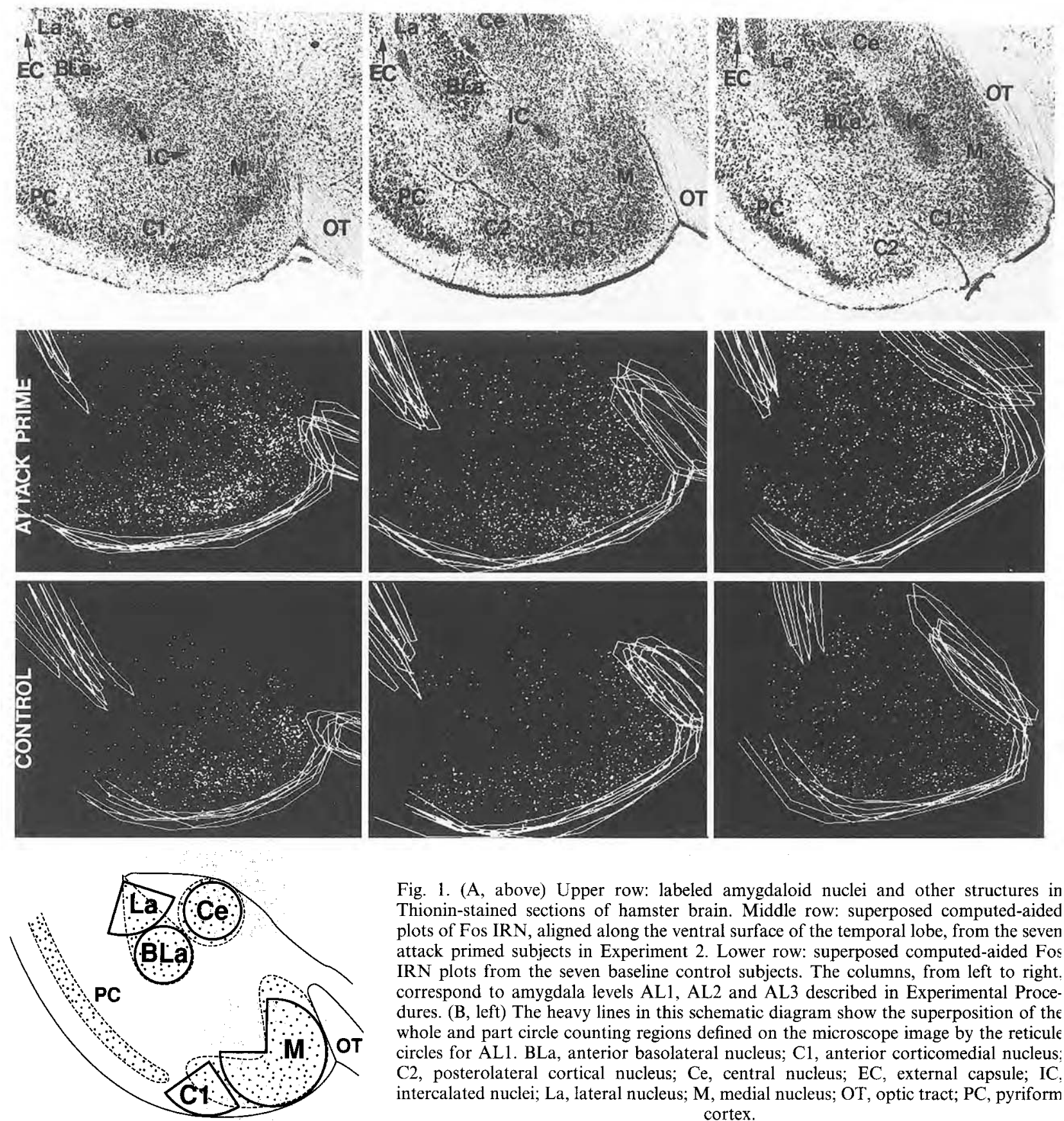

Fig. 1. (A, above) Upper row: labeled amygdaloid nuclei and other structures in Thionin-stained sections of hamster brain. Middle row: superposed computed-aided plots of Fos IRN, aligned along the ventral surface of the temporal lobe, from the seven attack primed subjects in Experiment 2. Lower row: superposed computed-aided Fos IRN plots from the seven baseline control subjects. The columns, from left to right, correspond to amygdala levels AL1, AL2 and AL3 described in Experimental Procedures. $(B$, left) The heavy lines in this schematic diagram show the superposition of the whole and part circle counting regions defined on the microscope image by the reticule circles for AL1. BLa, anterior basolateral nucleus; $\mathrm{Cl}$, anterior corticomedial nucleus; $\mathrm{C} 2$, posterolateral cortical nucleus; $\mathrm{Ce}$, central nucleus; EC, external capsule; IC intercalated nuclei; La, lateral nucleus; M, medial nucleus; OT, optic tract; PC, pyriform cortex.

duration measures were taken before the first attack on all probe trials. These contact durations were used as the measure of attack latency in Experiments 1 and 2; we have found that contact duration is proportional to the conventional elapsed time measure of latency (i.e. resident hamsters will spend about $75 \%$ of a 10 -min trial in contact with an intruder), but contact duration is a more reliable predictor of the priming effect than elapsed time. ${ }^{49}$ This is presumably because when the subject is at a distance from the intruder, it is out of contact with the attack-stimulating cues provided by the intruder. All training and testing was done during the dark phase of the light cycle. The minimum inter-test interval was two days.

Experiment 2. The aim of this experiment was to identify neural structures associated with attack priming. The 21 hamsters in Experiment 1 were matched into seven triplets based on their priming and probe attack latencies. One member of each triplet was attack primed (i.e. allowed a single attack upon an intruder) and the second exposed to a moving woodblock for a time equal to the attack latency of the primed animal (woodblock yoked control condition). The third subject was moved in its cage from cage rack to bench top as the other two subjects had been but was otherwise unperturbed (baseline control condition). Behavioral studies using Fos as a marker of brain activity entail the possibility that the gene expression is due to aspects of the stimulus situation and/or the animals' response other than those deemed important by the experimenter, e.g., behaviors occurring during the period between testing and perfusion. For this reason, just before each subject's final test, its cage was placed atop a Columbus Instruments 
Table 1. Mean areal densities \pm S.E.M. of Fos immunoreactive nuclei in amygdaloid nuclei of the three groups of subjects in Experiment 2

\begin{tabular}{lccc}
\hline & $\begin{array}{c}\text { Attack primed } \\
\text { (mean } \pm \text { S.E.M.) }\end{array}$ & $\begin{array}{c}\text { Yoked woodblock exposure } \\
\text { (mean } \pm \text { S.E.M.) }\end{array}$ & $\begin{array}{c}\text { Baseline control } \\
\text { (mean } \pm \text { S.E.M.) }\end{array}$ \\
\hline Regional densities of Fos-labeled nuclei (immunoreactive nuclei/mm ${ }^{2}$ ) & & \\
MN & $123.5 \pm 29.3^{*}$ & $77.8 \pm 20.8^{*}$ & $70.8 \pm 20.8^{*}$ \\
C1 & $108.2 \pm 28.1^{*}$ & $86.1 \pm 23.4^{*}$ & $81.4 \pm 28.8^{*}$ \\
C2 & $62.4 \pm 9.9$ & $57.6 \pm 15.0$ & $34.9 \pm 9.7$ \\
C3 & $96.8 \pm 25.1$ & $65.5 \pm 20.6$ & $49.5 \pm 19.8$ \\
Lateral nucleus & $10.7 \pm 2.0$ & $5.3 \pm 1.6$ & $10.0 \pm 7.0$ \\
Basolateral nucleus & $25.9 \pm 10.7$ & $12.4 \pm 6.5$ & $23.7 \pm 17.2$ \\
Amygdalo-hippocampal area & $61.8 \pm 19.8$ & $22.7 \pm 6.7$ & $36.8 \pm 22.0$ \\
Central nucleus $\dagger$ & 0.0 & 0.0 & 0.0 \\
\hline
\end{tabular}

*Significant difference among conditions at $P<0.02$.

$\dagger$ Median values.

activity meter to record activity in the $1 \mathrm{~h}$ between test and perfusion. Specific behaviors, including scrabbling at and jumping against the sides of the cage, climbing and clinging to the wire bars of the cage cover, rearing, grooming and digging, were also monitored for 3-min periods at $0,18,37$ and 57 min after testing. For every period, behaviors were scored as occurring (or not) within each of 12 consecutive 15-s segments (i.e. the maximum score for any behavior within a period was 12). At the end of the $1 \mathrm{~h}$ observation period, subjects were killed and prepared for Fos immunocytochemistry.

Experiment 3. This experiment examined changes in CMA c-fos expression associated with exposure to anesthetized intruders. Eight ovariectomized females drawn from the same population as the original 21 were paired on the basis of aggression screening scores. One member of each pair was attack primed, the other explored a pentobarbitalanesthetized intruder for $60 \mathrm{~s}$. They were killed $1 \mathrm{~h}$ after testing and prepared for Fos immunocytochemistry as in Experiment 1.

Experiment 4. This experiment evaluated the functional significance of the Fos localization by comparing the effects of CMA and control lesions on aggression and locomotion. Bilateral radiofrequency CMA lesions were made in 11 aggressive subjects prepared, maintained and selected as in Experiment 1 . To examine the effect of passing an electrode through the tissue lying dorsal to the CMA, electrode track puncture wounds were made in a second, control group $(n=7)$. Neocortical lesions were made in a third group to control for the effects of destroying a volume of brain tissue $(n=8)$. Beginning 12-14 days after surgery, subjects were given three priming/probe trial pairs using the procedure of Experiment 1 . The minimum interval between trial pairs was $48 \mathrm{~h}$. After aggression testing was completed, subjects were given two consecutive 10-min trials in a Wahmann running wheel. Between trials the wheel was stopped, the door to the wheel was opened, and the subject was allowed to exit into the wheel's antechamber and then to re-enter the wheel. Entry latency and wheel turns were recorded.

\section{RESULTS}

Experiment 1. Effects on subsequent attack latency of attack priming $v$ s the pursuit and biting of an inanimate object

This experiment yielded two important results. The first was that the priming effect was again replicated.
Attack latency on the priming trial was $164 \pm 19 \mathrm{~s}$ (mean \pm S.E.M.); latency on the probe trial, $111 \pm 28 \mathrm{~s}$, was reduced by $32 \%$. The second result was that pursuit and biting an inanimate object had little effect. Attack latency following interaction with the woodblock was $150 \pm 20 \mathrm{~s}$, only $9 \%$ less than the priming trial baseline. A log transformed, repeated measures ANOVA showed significant differences among the three conditions $\left(F_{2.40}=11.2\right.$, $P<0.0001)$. Post hoc Scheffé tests showed that this difference was due in part to the reduction in probe attack latency following the priming trial $\left(F_{2,40}=9.8\right.$, $P<0.001)$. Attack latencies following woodblock exposure did not differ from priming latencies (i.e. the $9 \%$ latency reduction following woodblock exposure was not significant; $F_{2,40}=0.3$ ), but these latencies were significantly greater than latencies to attack following a priming trial $\left(F_{2,40}=6.6\right.$, $P<0.005)$.

Experiment 2. Effects on $c$-fos expression in the amygdala of attack priming $v$ s the pursuit and biting of an inanimate object

Figure 1A compares composites of amygdala sections formed by superposing video images from the seven attack primed subjects to composites formed from the seven baseline controls. As this figure shows, c-fos expression proved to be a neuroanatomically localized marker of attack priming. Of the amygdaloid structures examined quantitatively, only the $\mathrm{MN}$ and the cortical nuclei showed differences among the three conditions which paralleled the shifts in attack latency in Experiment 1 (Table 1): relative to baseline controls, the mean areal density of Fos IRN in primed animals was increased $74 \%$ and $33 \%$ in $\mathrm{MN}$ and $\mathrm{Cl}$, respectively. There were increases of $10 \%$ and $6 \%$ in the respective mean Fos IRN areal densities of woodblock-exposed animals. The visual impression of differences among the behavioral conditions in Fos IRN areal density in the two areas was confirmed by ANOVA of the log 


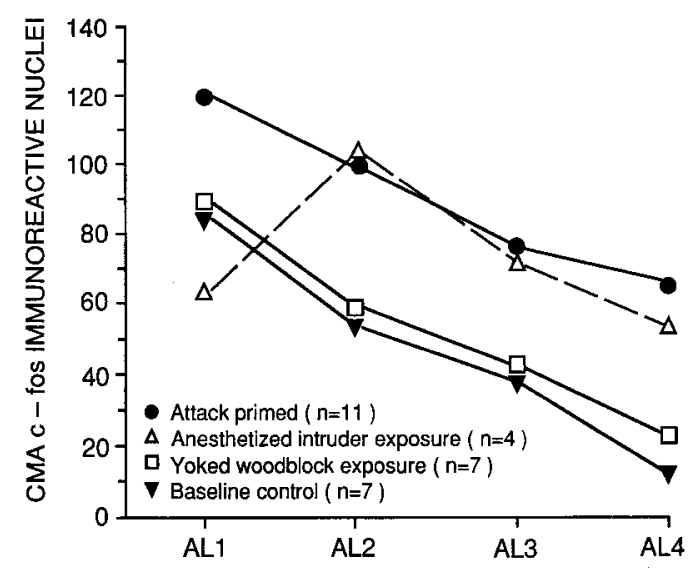

Fig. 2. Distributions of mean number of Fos IRN along the rostral caudal axis of the CMA in subjects which were attack primed, exposed to a woodblock or to an anesthetized intruder, or were unperturbed (Experiments 2 and 3 ). AL1-AL4 refer to the four rostrocaudal amygdala levels at which counts were made.

transformed data, which treated the matched triplets as repeated measures and used baseline rates of attack as a covariate $\left(F_{2,21}=4.9, P<0.02\right)$. No difference between $\mathrm{MN}$ and $\mathrm{Cl}$ was found $\left(F_{1,12}=0.04\right)$, so these structures were combined as the CMA for subsequent analysis. In contrast to its effects in the CMA, priming was not associated with substantial changes in Fos IRN in the lateral and basolateral nuclei of the amygdala. That the pattern of c-fos expression in these nuclei, attack primed $>$ baseline control $\gg$ woodblock exposed, was different from the pattern of effects on attack latencies is further evidence against the involvement of these structures in the priming effect, although the interpretation of this pattern is unclear at present. The central nucleus showed very few Fos IRN in any group (Table 1).

The pattern and magnitude of priming-induced CMA c-fos expression also appears to be behaviorally specific. First, CMA Fos IRN were more numerous in attack primed subjects than in subjects in the yoked woodblock condition (Table 1; also see Fig. 2), implying that the increased c-fos expression was not simply due to the localization, pursuit and biting of a salient object, but was specifically related to attack upon a conspecific. Secondly, there were no inter-group differences in grooming, scrabbling at the cage walls, or other behaviors in the hour between testing and perfusion. This eliminates the hypothesis that differences in CMA Fos IRN were due to differences in specific post-test behaviors (repeated measures ANOVA, $F_{2,12} \leq 2.5$, Table 2). During the post-test period, the primed animals were slightly, but not significantly, more active $\left(F_{2,12}=2.0\right.$; Table 2). More importantly, there were no significant within-group correlations between CMA Fos IRN and post-test activity (Table 2 ). General activity is an often used measure of non-specific arousal in rodents; to the extent that this measure is valid, it appears that the CMA c-fos expression induced by attack priming was unrelated to such arousal.

Experiment 3. Changes in corticomedial amygdala c-fos expression associated with exposure to an anesthetized intruder

The four additional primed animals showed the same pattern of Fos IRN at every anatomical level as the seven original ones $\left(F_{1,27}=0.4\right.$ between these two groups); their data were therefore pooled. As Fig. 2 shows, there were no differences in Fos IRN between intruder-exposed and attack primed animals at the three caudalmost levels of the CMA. This suggests that contact with the intruder is sufficient to activate caudal CMA neurons, even when an attack is not carried out. Vomeronasal and olfactory cues from the intruder are likely to have been the source of activation of caudal CMA neurons since the corticomedial nuclei are sites of these inputs (e.g., Refs 35 and 55). Such pheromonal cues release or facilitate aggression in many rodent species, including hamsters (e.g., Refs 20, 39 and 42). In contrast to the effects at caudal levels, the density of Fos IRN in the most anterior CMA section of the intruder-exposed subjects was at the baseline control value. This produced a statistically significant condition $x$ anatomical level interaction among the intruderexposed, attack primed and baseline control conditions $\left(F_{6,57}=8.2, P<0.0001\right)$. Pairwise post hoc tests revealed significant condition $\times$ anatomical level interactions for intruder-exposed vs baseline control animals and vs attack primed animals $\left(F_{3,57} \geq 4.8\right.$, $P<0.005$ ).

The finding that $60 \mathrm{~s}$ of exploration activates a large subset of CMA neurons which are activated by

Table 2. Mean activity counts and frequencies of behavior of subjects in the $1 \mathrm{~h}$ between test and killing in Experiment 2

\begin{tabular}{lcccrrr}
\hline B Attack primed & \multicolumn{3}{c}{ Yoked woodblock exposure } & \multicolumn{2}{c}{ Baseline control } \\
Behavior & Mean \pm S.E.M. & $r$ & Mean \pm S.E.M. & $r$ & Mean \pm S.E.M. & $r$ \\
\hline Behaviors occurring between test and killing and their correlation $(r)$ with CMA Fos IRN \\
Activity counts & $4439 \pm 599$ & 0.12 & $4001 \pm 445$ & -0.03 & $3510 \pm 699$ & -0.49 \\
Scrabble/jump/hang on bars & $4.6 \pm 1.9$ & 0.03 & $5.6 \pm 0.9$ & 0.11 & $3.6 \pm 1.2$ & -0.40 \\
Rear & $4.3 \pm 0.8$ & 0.13 & $4.3 \pm 0.6$ & -0.11 & $4.1 \pm 0.6$ & -0.73 \\
Groom & $4.8 \pm 1.4$ & -0.49 & $4.1 \pm 0.8$ & -0.36 & $1.6 \pm 0.5$ & -0.28 \\
Dig & $1.5 \pm 0.3$ & 0.50 & $3.9 \pm 1.1$ & -0.33 & $3.3 \pm 1.2$ & -0.28 \\
\hline
\end{tabular}



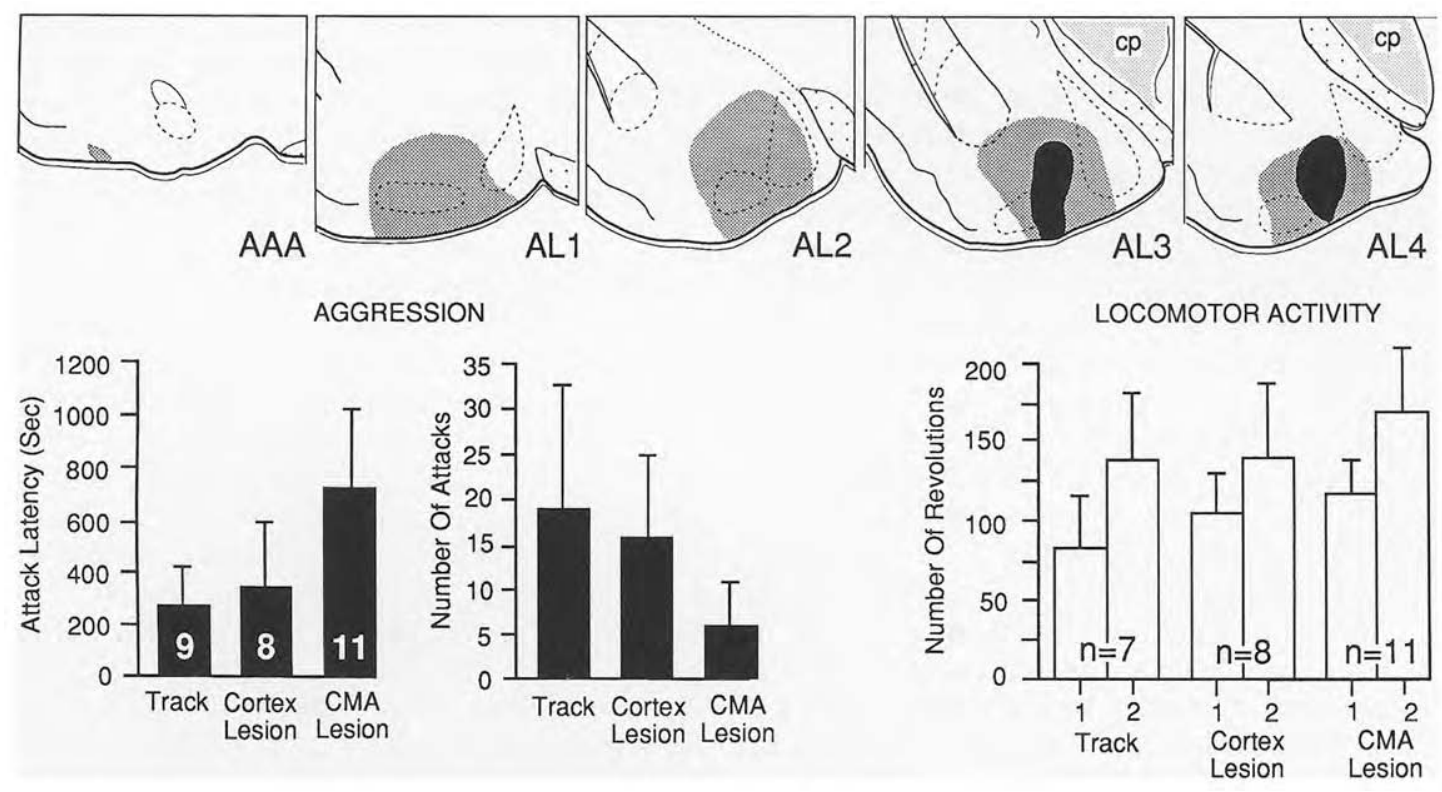

Fig. 3. Representative CMA lesions (upper row) and lesion effects (lower row) in Experiment 4. Lesions were usually slightly larger on one side of the brain than the other. The stippled area in the sections represents the side with the larger lesion in the subject whose total lesion volume ranked third out of 11 and whose lesion centroid was the third most rostral. The black area represents the larger lesion in the subject whose lesion ranked ninth in volume and eighth in rostrocaudal location. Embedded in the bars in the lower row are the number of subjects/group. The double bars on the right side, labeled 1 and 2 , represent the first and second $10-$ min trials in the running wheel, respectively. Error bars are S.E.M.

attack priming is entirely consistent with the behavioral observation that this exposure reduces subsequent attack latency almost to the attack primed level. From the lack of increase in c-fos expression in the rostral CMA, it may be inferred that neurons there may initiate, and/or receive feedback from, overt attack. Such feedback could be internal (e.g., somatosensory/autonomic signals generated by the act of biting) or external (e.g., vocalization by the intruder as a consequence of being bitten).

Experiment 4. Effects of corticomedial amygdala and control lesions on aggression and locomotion

As shown in Fig. 3, lesions of the CMA significantly reduced the number of attacks $\left(F_{2,24}=5.4\right.$, $P<0.02)$. Eight of the 11 CMA subjects completely failed to attack on at least one pair of trials. When animals fail to attack, contact duration as a latency measure becomes difficult to interpret. Therefore, elapsed time measures of latency were compared in this experiment; trials without attack were assigned a latency of $1201 \mathrm{~s}$. CMA lesions significantly increased attack latency $\left(F_{2,24}=10.7, P<0.001\right)$ relative to control groups (see Fig. 3). A more detailed understanding of these effects was provided by plotting the lesions on standard section drawings at predetermined AP levels through the amygdala. As shown by the representative lesions in Fig. 3, damage was distributed along the rostrocaudal extent of the amygdala. Lesion volume was estimated by summing the planimetrically determined areas of damage at each level; the lesion centroid was then determined. There was a correlation between lesion position and effects on aggression: the more anterior the centroid, the longer the mean elapsed time to the first bite on either trial (Pearson $r=-0.71, P<0.02$ ). The relationship between lesion site and attack latency was confounded by the fact that the more anterior lesions were larger. However, the correlation between lesion volume and attack latency was non-significant $(r=0.32)$, and a partial correlation between lesion site and attack latency which removed the effect of lesion size remained significant $(r=-0.69$, $P<0.02$ ).

These findings are consistent with earlier studies showing that CMA lesions reduce intraspecific aggression in hamsters, ${ }^{8,58,61}$ rats (e.g., Refs 38 and 64; cf. Refs 23, 24 and 57) and mice. ${ }^{22}$ We note that our differentiation between the effects of neocortical and CMA lesions does not exclude the logical possibility that lesions of other limbic structures might also reduce aggression, thus weakening the claim of neuroanatomical specificity. However, an earlier study carried out under similar conditions in our laboratory did not find any reductions in attack latency in hamsters with septal lesions $(n=7)$ compared to those with neocortical lesions $(n=5$; data not shown). Furthermore, a number of the studies just cited found that CMA lesions had greater effects on aggression than lesions of other limbic structures outside the amygdala (e.g., Ref. 58) or other nuclei within the amygdala (e.g., Ref. 38). Finally, still other studies have found little effect on intraspecific 
aggression of large amygdala lesions which spare the CMA. $^{3,9}$

In contrast to effects on latency to attack, CMA lesions in our study did not affect first or second latency to enter the running wheel $\left(F_{2,23}=1.3\right)$ and there was no significant correlation within the CMA group between entry latency and lesion site or size $(r \leq 0.43)$. There were also no differences between groups in the number of wheel turns $\left(F_{2,23}=1.6\right.$; see Fig. 3). Hovever, all animals in both control groups and 10 of the 11 CMA animals ran more in the second trial. This produced a highly significant locomotor practice effect $\left(F_{1,23}=22.2, P<0.0001\right)$. A lack of trial $\times$ group interaction $\left(F_{2,23}=0.8\right)$ indicated that this practice effect occurred equally strongly in all groups. Overall, these findings indicate that the CMA lesions did not reduce aggression by increasing general timidity (latency to enter the wheel was unaffected), or by interfering with locomotor ability (wheel turning was unaffected), or by affecting the facilitation of behavior through previous experience (the increase in wheel turns from first to second trials was unaffected).

Further results - Towards a quantitative neurobehavioral model of aggressive arousal

The results of Experiment 4, showing that anterior CMA lesions reduce aggression, complement the finding that sniffing an anesthetized target activates only the caudal CMA (Experiment 3). Taking all of the foregoing results together, we infer three rules linking attack priming with neuronal activation in the CMA as that is marked by c-fos expression.

(1) Exposure to aggression-facilitating olfactory/ vomeronasal cues increases neuronal activation in the caudal CMA. Exposure to stimuli which release attack (e.g., movement of the intruder) activates the rostral CMA; this effect sums with or is facilitated by caudal CMA activation, possibly through rostrally directed projections within the CMA. ${ }^{13,25,26,41}$

(2) The level of activation in the rostral CMA controls the instantaneous probability of attack: the greater the activation at a given moment, the higher the probability of attack at that moment.

(3) The activation of these neurons (or their increased potential for re-activation) persists for a period of time following the first attack, thus accounting for the priming effect.

Rule 1 is supported by the convergence of two estimates of the mean rate of increase in Fos IRN during exposure to an intruder. The mean rate of Fos IRN increase in the three caudal CMA levels calculated from the difference between the four subjects which were exposed to anesthetized intruders for $60 \mathrm{~s}$ and the seven baseline control subjects was $35.1 / \mathrm{mm}^{2} / \mathrm{min}$. The mean rate of increase derived from the regression of Fos IRN on attack latency in the 11 attack primed subjects was $21.4 / \mathrm{mm}^{2} / \mathrm{min}$.

Rule 2 is a theoretical proposition which lies at the heart of the model being developed. It is based, in part, on a parallel between the increases in CMA Fos IRN reported here and a novel $\theta(t)$ transformation of survival analysis, which has shown that the instantaneous probability of attack is low at the beginning of a trial and increases to an asymptote during the course of the trial. ${ }^{49}$ Rule 1 dictates that Fos IRN will increase during continuing contact with the intruder, while rule 2 implies that the latency to attack will shorten as Fos IRN increases. Evidence for these opposing tendencies was obtained from a multiple regression of CMA Fos IRN in primed subjects on their final (pre-perfusion) attack latencies (a close estimate of their duration of contact with the intruder) and on their mean attack latency on trials in the days preceding the perfusion (a measure of their mean probability of attack ${ }^{49}$ ). The multiple regression was significant $\left(r^{2}=0.69, F_{2,8}=8.9, P<0.01\right)$. As inferred, CMA Fos IRN was positively correlated with the latency of the attack just preceding perfusion (the regression coefficient $\beta=0.83, t=3.9$, $P<0.005$ ), but negatively correlated to the mean attack latency in the preceding days $(\beta=-0.25)$.

This confirmation of oppositely directed correlations has encouraged us to develop a quantitative neurobehavioral model in which rules 1 and 2 are applied using a suitably chosen growth function to represent the increase of Fos IRN with time. This effort has shown that the two rules need not cancel each other out, but that their interaction produces different expected relationships between attack latency and Fos IRN depending upon the growth function selected and its assumed distribution across subjects. Our most satisfactory model to date, which characterizes differences among individual subjects as differences in a single parameter of the growth function, reproduces the observed relationship between attack latency and Fos IRN counts in some detail and accounts for a substantial proportion of the variance in this relationship (Potegal and Coombes, in preparation).

The $\theta(t)$ analysis noted above has suggested that the critical change accounting for the priming effect is an increase in the rate at which attack probability reaches an asymptote on the probe trial. ${ }^{49}$ Rule 3 is a statement that the processes underlying the more rapid rise in attack probability persist for a period of time following the priming trial. The extended timecourse of c-fos expression is consistent with this rule. High-frequency stimulation of the CMA induces a delayed and protracted reduction in attack latency. ${ }^{51}$

\section{DISCUSSION}

\section{Aggression and sex}

Experiments 2 and 3 demonstrated that CMA c-fos expression is increased by attack priming and by 
exposure to an anesthetized conspecific, two situations which induce aggressive arousal. In contrast, no increase followed sensorimotor acts similar to those of attack priming which do not elicit aggressive arousal. These observations are consistent with the hypothesis that CMA activation is associated with aggressive arousal, but leave open the possibility that other social interactions, such as copulation, might also be associated with CMA activation. There are, in fact, recent reports of increased CMA c-fos expression associated with copulation in male rats and hamsters, ${ }^{2,28,54}$ and estradiol administration in female rats. ${ }^{21}$ These observations might appear to bring into question the specificity of the association between CMA activation and aggressive behavior. However, careful examination of the figures and tables in these papers shows that increases in c-fos expression are found most clearly or only in the more dorsal and caudal aspects of the CMA. Several lines of evidence have implicated these subregions of the CMA in the control of sexual behavior; for example, these are the regions containing the highest density of sex steroid-concentrating neurons. $^{30}$ Our observations on the CMA's involvement in aggression have, in contrast, focused on the rostral and ventral aspects of the CMA. This contrast was highlighted by a preliminary study carried out in our laboratory using four pairs of animals selected from our population of aggressive, ovariectomized females. ${ }^{50}$ Both members of each pair were pretreated with $30 \mu \mathrm{g}$ of i.m. estradiol benzoate in oil 48 and $24 \mathrm{~h}$ before testing, and $500 \mu \mathrm{g}$ of progesterone $4 \mathrm{~h}$ before testing. One member of each pair interacted with a stud male placed in her home cage until she showed lordosis, the other underwent no behavioral manipulation. Measurements of the type used in Experiments 2 and 3 showed overall levels of c-fos expression that were lower in these animals than in animals which had been attack primed. More telling was the observation that in the subjects in which lordosis had been elicited by a male, the number of c-fosexpressing neurons in the CMA increased in successively more caudal planes, a pattern different from that shown in Fig. 2. We are by no means suggesting that aggressive and sexual behaviors involve distinct and isolated regions of the CMA. The many connections and interactions between aggressive and sexual behavior demonstrated in a multitude of studies instead suggest gradients of influence. We conjecture that, at least in female hamsters, the ventral and rostral CMA may exert a greater or more direct control on aggression and a lesser or indirect influence on copulation, while a different pattern of control is obtained for the dorsal and caudal CMA. One possible caveat to this conjecture is the possibility that limbic system function in the female subjects of the present study may have been altered by their chronic ovariectomy. ${ }^{37}$ The study of changes in CMA c-fos expression associated with aggressive and sexual priming in gonadally intact females and males would be an important extension of the present work.

Output pathways from the corticomedial amygdala and the variables that might be controlled by those pathways

The results reported here raise the question of the output pathways through which the CMA influences behavior. The major projections of the CMA are to hypothalamic nuclei, including the ventrolateral aspect of the ventromedial nucleus and the adjacent perifornical area, ${ }^{13,25,26,31,32,40,41}$ these are areas in which electrical stimulation evokes attack in rats. ${ }^{33}$ MacDonnell and Flynn's ${ }^{36}$ classic finding, that electrical stimulation of the lateral hypothalamus which induced attack in cats also activates an otherwise latent biting reflex in response to a touch on the lips, has been extended to effects on the cutaneous sensory fields that trigger paw strikes and on prey tracking eye movements (for review see Ref. 33). The existence of a hypothalamically modulated biting reflex has been replicated in rats. ${ }^{60}$ Rule 2 of the neurobehavioral model asserts that increased neuronal activation of the CMA is associated with increased probability of attack, but does not specify the mechanisms by which this is accomplished. We now propose that the CMA controls the instantaneous probability of attack by modulating hypothalamic sensorimotor functions. This conjecture is consistent with findings that stimulation of medial amygdaloid nuclei facilitates hypothalamically elicited attack (e.g., Ref. 4).

\section{Time-course and redirection of aggressive arousal}

The analysis just described focuses on processes occurring during the first few minutes of contact between subject and intruder. However, a salient feature of the priming paradigm is that the aggressive arousal so induced has been demonstrated to persist in time. Thus, physiological mechanisms found to be associated with attack priming become, ipso facto, candidates for projecting aggressive arousal into the future. In both female hamsters and male rats, the persistence of the priming effect can be modeled as the addition of two processes. ${ }^{46}$ The first process is maximal immediately after the priming attack and decays away with a half-life of the order of several minutes. The second process grows slowly, is manifest at about $30 \mathrm{~min}$ and declines within a few hours after that. The latter process is roughly coincident with the appearance of Fos protein (e.g., Ref. 56), suggesting the possibility that downstream genomic effects of c-fos expression in the CMA might contribute to the prolonged time-course of attack priming. We also note the temporal coincidence in the report of Payne et al. ${ }^{43}$ that hypothalamic serotonin concentration in hamsters reaches a nadir $30 \mathrm{~min}$ after a fight. 
The attack priming effect is not restricted to female hamsters; we have also demonstrated it in male hamsters ${ }^{18}$ and rats. ${ }^{46}$ Other laboratories have reported the phenomenon in mice ${ }^{34,62}$ and fighting fish. ${ }^{5,15}$ In the broader context of agonistic behavior, the persistence of CMA-mediated aggressive arousal might be the basis for such phenomena as the redirection of aggression in which the arousal engendered in a fight between two animals carries over to an attack on a third, previously uninvolved animal. Indeed, the attack priming paradigm can be viewed as a formal model of redirection. Redirection of aggression has been observed in rodents, ${ }^{1,11,59}$ but is more often reported in primates. ${ }^{10}$ This raises the question of the cross-species generality of our findings. Olfactory and vomeronasal inputs have a major control over aggression in rodents; does the CMA retain a crucial role in the aggressive arousal of microsmatic species where olfactory/vomernasal stimuli are of lesser importance? Expressions and gestures of threat and submission in monkeys, for example, are communicated visually. It is thus of interest that medial amygdala neurons in rhesus monkeys are not only responsive to visual stimuli, they are attuned to facial expression (e.g., Ref. 6), suggesting that the CMA may play a role in mediating agonistic interactions in this species as well.

\section{CONCLUSIONS}

C-fos expression, used as a marker of endogenous neural activity in the context of the attack priming paradigm, has allowed us to identify the CMA as a candidate region in the mediation of aggressive arousal in female hamsters. The subsequent experimental manipulation, making lesions in the CMA, has confirmed this region as critical in the mediation of aggression. Behavioral controls and neuroanatomical comparisons in both stages of the research support the specificity of the results. The aggressive arousal induced by attack priming has been demonstrated to persist in time. Thus, the CMA activation preceding and accompanying a priming attack may be at least as much associated with an animal's probable aggressive behavior in its immediate future as with its behavior in the recent past. The time-course of c-fos expression is consistent with the persistence of change in attack probability that follows attack priming.

\section{REFERENCES}

1. Alberts J. R. and Galef B. G. Jr (1973) Olfactory cues and movement; stimuli mediating intraspecific aggression in the wild Norway rat. J. comp. physiol. Psychol. 85, 233-242.

2. Baum M. J. and Everitt B. J. (1992) Increased expression of c-fos in the medial preoptic area after mating in male rats: role of afferent inputs from the medial amygdala and midbrain central tegmental field. Neuroscience 50, 627-646.

3. Blanchard D. C. and Takahashi S. N. (1988) No change in intermale aggression after amygdala lesions which reduce freezing. Physiol. Behav. 42, 613-616.

4. Block C. H., Siegel A. and Edinger H. (1980) Effects of amygdaloid stimulation upon the trigeminal sensory fields of the lip that are established during hypothalamically-elicited quiet biting attack. Brain Res. 197, 39-55.

5. Bronstein P. (1989) The priming and retention of agonistic motivation in male Siamese fighting fish, Betta splendens. Anim. Behav. 37, 165-166.

6. Brothers L., Ring B. and Kling A. (1990) Response of neurons in the macaque amygdala to complex social stimuli. Behav. Brain Res. 41, 199-213.

7. Brown R. E. (1985) The rodents. II. Suborder Myomorpha. In Social Odours in Mammals (eds Brown R. E. and Macdonald D. W.), Vol. 1, pp. 345-457. Clarendon Press, Oxford.

8. Bunnell B. N., Sodetz J. and Shalloway D. I. (1970) Amygdaloid lesions and social behavior in the golden hamster. Physiol. Behav. 5, 153-161.

9. Busch D. E. and Barfield R. J. (1974) A failure of amygdaloid lesions to alter agonistic behavior in the laboratory rat. Physiol. Behav. 12, 887-892.

10. Cheney D., Seyfarth R. and Smuts B. (1986) Social relationships and social cognition in nonhuman primates. Science 234, 1361-1366.

11. Eisenberg J. F. (1962) Studies on the behavior of Peromsycus maniculatus gambelli and Peromyscus californicus parasiticus. Behaviour 19, 177-207.

12. Floody O. and Pfaff D. (1977) Aggressive behavior in female hamsters: the hormonal basis for fluctuations in female aggressiveness correlated with estrous state. J. comp. physiol. Psychol. 91, 443-464.

13. Gomez D. M. and Newman S. W. (1992) Differential projections of the anterior and posterior regions of the medial amygdaloid nucleus in the Syrian golden hamster. J. comp. Neurol. 317, 195-218.

14. Grant E. C. and MacKintosh J. H. (1963) A comparison of the social postures of some common laboratory rodents. Behaviour 21, 246-259.

15. Halperin J. R. P., Dunham D. W. and Ye S. (1992) Social isolation increases social display after priming in Betta splendens but decreases aggressive readiness. Behav. Processes 28, 13-32.

16. Hammond R, and Rowe F. (1976) Medial preoptic and anterior hypothalamic lesions: influences on aggressive behavior in female hamsters. Physiol. Behav. 17, 507-513.

17. Hancock M. B. (1986) Two color immunoperoxidase staining: visualization of anatomic relationships between immunoreactive neural elements. Am. J. Anat. 175, 343-352.

18. Hebert M., Potegal M. and Meyerhoff J. (1994) Flight-elicited attack and priming of aggression in "non-aggressive" hamsters. Physiol. Behav. 56, 671-675.

19. Heiligenberg W. (1974) Processes governing behavioral states of readiness. In Advances in the Study of Behavior (eds Lehrman D. S., Rosenblatt J. S., Hinde R. A. and Shaw F.), Vol. V, pp. 173-200. Academic Press, New York. 
20. Ingersoll D. W., Morley K., Benvenga M. and Hands C. (1986) An accessory sex gland aggression-promoting chemosignal in male mice. Behav. Neurosci. 100, 777-782.

21. Insel T. (1990) Regional induction of c-fos-like protein in rat brain after estradiol administration. Endocrinology 126, $1849-1853$.

22. Kemble E. D. (1981) Some behavioral effects of amygdaloid lesions in Northern grasshopper mice. In The Amygdaloid Complex (ed. Ben-Ari Y.), IRSERM Symposium No. 20, pp. 305-315. Elsevier/North Holland Press, Amsterdam.

23. Kemble E. D., Blanchard D. C. and Blanchard R. J. (1990) Effects of regional amygdaloid lesions on flight and defensive behaviors of wild black rats (Rattus rattus). Physiol. Behav. 48, 1-5.

24. Kemble E. D., Blanchard D. C., Blanchard R. J. and Takushi R. (1984) Taming in wild rats following medial amygdaloid lesions. Physiol. Behav. 32, 131-134.

25. Kevetter G. A. and Winans S. (1981) Connections of the corticomedial amygdala in the golden hamster. I. Efferents of the vomeronasal amygdala. J. comp. Neurol. 197, 81-98.

26. Kevetter G. A. and Winans S. (1981) Connections of the corticomedial amygdala in the golden hamster. II. Efferents of the olfactory amygdala. J. comp. Neurol. 197, 99-111.

27. Koh E. T., Stopa E. G., King J. C., Reynolds B. R., Rogers W. T., Schwaber J. S. and Hillman D. E. (1989) Computer aided mapping of specific neural populations in the human brain. Biotechniques 7, $596-602$.

28. Kollack S. S. and Newman S. W. (1992) Mating behavior induces selective expression of Fos protein within the chemosensory pathways of the male Syrian hamster brain. Neurosci. Lett. 143, 223-228.

29. Krames L. and Shaw B. (1973) Role of previous experience in the male rat's reaction to odors from group and alien conspecifics. J. comp. physiol. Psychol. 82, 444448.

30. Krieger M. S., Morrell J. I. and Pfaff D. W. (1976) Autoradiographic localization of estradiol-containing cells in the female hamster. Neuroendocrinology 22, 193-205.

31. Krettek J. E. and Price J. L. (1978) Amygdaloid projections to subcortical structures within the basal forebrain and brainstem in the rat and cat. J. comp. Neurol. 178, 225-254.

32. Krettek J. E. and Price J. L. (1978) A description of the amygdaloid complex in the rat and cat with observations on intraamygdaloid axonal connections. J. comp. Neurol. 178, 255-280.

33. Kruk M. R. (1991) Ethology and pharmacology of hypothalmic aggression in the rat. Neurosci. Biobehav. Rev. 15, $527-538$.

34. Lagerspetz K. (1969) Aggression and aggressiveness in laboratory mice. In Aggressive Behavior (eds Garattini S. and Sigg E. B.), pp. 77-85. John Wiley, New York.

35. Lehman M. N. and Winans S. S. (1982) Vomeronasal and olfactory pathways to the amygdala controlling male hamster sexual behavior: autoradiographic and behavioral analyses. Brain Res. 240, 27-41.

36. MacDonnell M. F. and Flynn J. P. (1966) Control of sensory fields by stimulation of the hypothalamus. Science 152, $1406-1408$

37. Meisel R. L. and Luttrell V. R. (1990) Estradiol increases the dendritic length of ventromedial hypothalamic neurons in female Syrian golden hamsters. Brain Res. Bull. 25, 165-168.

38. Miczek K. A., Bryzkynski T. and Grossman S. P. (1974) Differential effects of lesions in the amygdala, periamygdaloid cortex, and stria terminalis on aggressive behaviors in the rat. J. comp. physiol. Psychol. 87, 760-771.

39. Murphy M. (1976) Olfactory stimulation and olfactory bulb removal: effects on territorial aggression in male Syrian golden hamsters. Brain Res. 113, 95-110.

40. Ono T., Luiten P. G. M., Nishijo H., Fukuda M. and Nishino H. (1985) Topographic organization of projections from the amygdala to the hypothalamus of the rat. Neurosci. Res. 2, 221-239.

41. Ottersen O. P. (1980) Afferent connections to the amygdaloid complex of the rat and cat: II. Afferents from the hypothalamus and the basal telencephalon. J. comp. Neurol. 194, 267-289.

42. Payne A. P. (1974) The effects of urine on aggressive responses by male golden hamsters. Aggressive Behav. 1, 71-80.

43. Payne A. P., Andrews M. J. and Wilson C. A. (1984) Housing, fighting, and biogenic amines in the midbrain and hypothalamus of the golden hamster. In Ethopharmacological Aggression Research (eds Miczek K., Kruk M. and Olivier B.), pp. 227-248. Alan R. Liss, New York.

44. Potegal M. (1984) The persistence of attack satiation in female golden hamsters. Aggressive Behav. 10, 303-307.

45. Potegal M. (1991) Attack priming and satiation in female golden hamsters: tests of some alternatives to the aggression arousal interpretation. Aggressive Behav. 17, 327-336.

46. Potegal M. (1992) Time course of aggressive arousal in female golden hamsters and male rats. Behav. neural Biol. 58, $120-124$

47. Potegal M. (1992) Aggression and aggressiveness in female golden hamsters. In Of Mice and Women: Aspects of Female Aggression (eds Bjorkqvist K. and Niemela P.), pp. 329-350. Academic Press, San Diego.

48. Potegal M., Blau A., Black M. and Glusman M. (1980) A technique for the study of intraspecific aggression in the golden hamster under conditions of reduced target variability. Psychol. Rec. 30, 191-200.

49. Potegal M. and Coombes K. (1995) Attack priming and aggressive arousal in female Syrian golden hamsters, Mesocricetus auratus. Anim. Behav. 49, 931-947.

50. Potegal M., Ferris C. and Skaredoff L. (1991) The corticomedial amygdala and hamster agonistic behavior. Soc. Neurosci. Abstr. 17, 877.

51. Potegal M., Herbert M., DeCoster M. and Meyerhoff J. (1996) Brief, high frequency stimulation of the corticomedial amygdala induces a delayed and prolonged increase of aggressiveness in male Syrian golden hamsters. Behav. Neurosci. 110, 401-412.

52. Potegal M. and Popken J. (1985) The time course of attack priming effects in female golden hamsters. Behav. Proc. 11, 199-208.

53. Potegal M. and tenBrink L. (1984) Behavior of attack-primed and attack-satiated female golden hamsters (Mesocricetus auratus). J. comp. Psychol. 98, 66-75.

54. Robertson G. S., Pfaus J. G., Atkinson L. J., Matsumura H., Phillips A. G. and Fibiger H. C. (1991) Sexual behavior increases c-fos expression in the forebrain of the male rat. Brain Res. 564, 352-357.

55. Scalia F. and Winans S. (1975) The differential projections of the olfactory bulb and accessory olfactory bulb in mammals. J. comp. Neurol. 161, 31-56. 
56. Sheng M. and Greenberg M. E. (1990) The regulation and function of c-fos and other intermediate early genes in the nervous system. Neuron 4, 477-485.

57. Shibata S., Yamamoto T. and Ueki S. (1982) Differential effects of medial, central, and basolateral amygdaloid lesions on four models of experimentally-induced aggression in rats. Physiol. Behav. 28, 289-294.

58. Shipley J. E. and Kolb B. (1977) Neural correlates of species-typical behavior in the Syrian golden hamster. J. comp. physiol. Psychol. 91, 1056-1073.

59. Simmel E. C. and Walker D. A. (1970) Social priming for agonistic behavior in a "docile" mouse strain. Am. Zool. 10, 486487.

60. Smith D. (1972) Increased perioral responsiveness: a possible explanation for the switching of behavior observed after lateral hypothalamic stimulation. Physiol. Behav. 8, 617-621.

61. Takahashi L. K. and Gladstone C. D. (1988) Medial amygdaloid lesions and the regulation of sociosexual behavioral patterns across the estrous cycle in female golden hamsters. Behav. Neurosci. 102, 268-275.

62. Tellegen A. and Horn J. M. (1972) Primary aggressive motivation in three inbred strains of mice. J. comp. physiol. Psychol. 78, 279-304.

63. Vandenbergh J. G. (1971) The effects of gonadal hormones on the aggressive behavior of adult golden hamsters. Anim. Behav. 19, 589-594.

64. Vochteloo J. D. and Koolhaas J. M. (1987) Medial amygdala lesions in male rats reduce aggressive behavior: interference with experience. Physiol. Behav. 41, 99-102. 